\title{
CONTRIBUIÇÃO À IDENTIFICAÇÃO E DISTRIBUIÇÃO DAS SUBESPÉCIES DE LUTREOLINA CRASSICAUDATA (DESMAREST) (MARSUPIALIA, MAMMALIA)
}

\author{
Maurício Graipel ${ }^{1}$ \\ Paul Richard M. Miller ${ }^{2}$ \\ Alfredo Ximenez ${ }^{1}$
}

\begin{abstract}
CONTRIBUITION TO THE IDENTIFICATION AND DISTRIBUTION OF SUBESPECIES of LUtreolina CRaSSicaudata (Desmarest) (Marsupialia, Mammalia). The subespecie Lutreolina crassicaudata paranalis Thomas, 1923 is validated through analysis of variance of 13 craniometrical measurements. There is significant separation for sex in 10 of 13 craniometrical measurements. The possibility of validation of subespecies L. c. bonaria Thomas, 1923 through coloration variation is discussed.

KEY WORDS. Distribution, subespecies, craniometrical measurements analysis, skin, sexual dimorfism.
\end{abstract}

Lutreolina crassicaudata (Desmarest, 1804) é um Didelphidae que, apesar de habitar locais urbanizados e de fácil acesso, possui comportamento pouco conhecido. No Brasil recebe o nome vernacular de "cuíca" que também é aplicado a outras espécies de marsupiais de porte médio.

A espécie apresenta uma distribuição disjunta, ocorrendo no Paraguai, Uruguai, oeste dos Andes na Bolívia, e nordeste da Argentina; no Brasil ao sul do Mato Grosso do Sul, São Paulo, Rio de Janeiro, Paraná, Rio Grande do Sul, e Santa Catarina. Ao norte da América do Sul, ocorre na Guiana, leste da Venezuela e Colômbia (e.g. MARSHALl 1978; NowAK \& PARAdiso 1983; MonTEIRo FiLHO \& DIAS 1990).

A história taxonômica da espécie é descrita por THOMAS (1923), CABRERA (1957), XiMENEZ (1967) e MARSHALL (1978), onde são relacionadas e discutidas as sub-espécies.

O objetivo deste trabalho é revalidar, através de análise de variância de medidas craniométricas e observações de materiais mantidos em museus, a subespécie L. c. paranalis Thomas, 1923, ssp. rev..

1) Departamento de Ecologia e Zoologia, Centro de Ciências Biológicas, Universidade Federal de Santa Catarina. 88040-970 Florianópolis, Santa Catarina, Brasil.

2) Departamento de Engenharia Rural, Centro de Ciências Agrárias, Universidade Federal de Santa Catarina. Rodovia SC 402 Km 3 1346, 88040-900 Florianópolis, Santa Catarina, Brasil. 


\section{MATERIAL E MÉTODOS}

Para análise de dados foram consideradas as medidas dos crânios de espécimens adultos sexados e de procedência comprovada. Foram considerados adultos os animais com dentição definitiva, com fórmula dentária: I 5/4, C 1/1, P 3/3 e M $4 / 4$.

Os dados foram coletados nas seguintes instituições: Museu Nacional, Rio de Janeiro (MNRJ); Museu de Zoologia da Universidade de São Paulo, São Paulo (MZUSP); Museu de História Natural da Universidade Estadual de Campinas, São Paulo (ZUEC); Museu de História Natural Capão da Imbuia (MHNCI) Curitiba, Paraná; Coleção Científica de Mamíferos da Universidade Federal de Santa Catarina, Florianópolis (UFSC); e Museu de Ciências Naturais da Fundação Zoobotânica do Rio Grande do Sul, Porto Alegre (FZBRS) (Tab. I).

Tabela I. Localidades de coleta dos exemplares e instituição depositária (peles e crânios).

\begin{tabular}{|c|c|c|}
\hline Localidade & Instituição & $\begin{array}{l}\text { Número de exemplares } \\
\text { da amostra (total) }\end{array}$ \\
\hline \multicolumn{3}{|l|}{ BRASIL } \\
\hline \multicolumn{3}{|l|}{ Mato Grosso do Sul } \\
\hline Maracajú & MNRJ & (2) \\
\hline \multicolumn{3}{|l|}{ Grupo 1} \\
\hline São Paulo & MZUSP & (1) \\
\hline \multirow[t]{2}{*}{ Americana } & MNRJ & $4(4)$ \\
\hline & ZUEC & $(4)$ \\
\hline Américo Brasiliense & ZUEC & (1) \\
\hline \multirow[t]{2}{*}{ Campinas } & MNRJ & (5) \\
\hline & ZUEC & $2(3)$ \\
\hline Emas * & MZUSP & (1) \\
\hline Franca & MZUSP & $1(1)$ \\
\hline Itaporanga & MZUSP & $(1)$ \\
\hline Nova Odessa * & MZUSP & $1(1)$ \\
\hline Piracicaba & ZUEC & $(1)$ \\
\hline Pirassununga & MZUSP & (1) \\
\hline \multirow[t]{2}{*}{ Paulínia } & MZUSP & (1) \\
\hline & ZUEC & (1) \\
\hline Ribeirăo Bonito & MZUSP & $1(1)$ \\
\hline Ribeirão Preto & MZUSP & $1(7)$ \\
\hline Tapiratuba (Morro Agudo) & MZUSP & (1) \\
\hline \multicolumn{3}{|l|}{ Grupo 2} \\
\hline \multicolumn{3}{|l|}{ Paraná } \\
\hline Curitiba & $\mathrm{MHNCl}$ & (1) \\
\hline Ilha Grande & $\mathrm{MHNCl}$ & $1(1)$ \\
\hline \multicolumn{3}{|l|}{ Santa Catarina } \\
\hline Florianópolis & UFSC & $18(29)$ \\
\hline Rio Grande do Sul & MZUSP & (1) \\
\hline Porto Alegre & FZBRS & (1) \\
\hline Rio Grande & FZBRS & (1) \\
\hline \multirow[t]{3}{*}{ São Lourenço } & MZUSP & $1(6)$ \\
\hline & MNRJ & (1) \\
\hline & & (continua) \\
\hline
\end{tabular}


Tabela I. Continuação.

\begin{tabular}{|c|c|c|}
\hline Localidade & Instituição & $\begin{array}{c}\text { Número de exemplares } \\
\text { da amostra (total) }\end{array}$ \\
\hline \multicolumn{3}{|l|}{ Grupo 3} \\
\hline \multicolumn{3}{|l|}{ ARGENTINA } \\
\hline \multicolumn{3}{|l|}{ Buenos Aires } \\
\hline Arroyo Pereira & MNHN & $1(1)$ \\
\hline Campaña & MNRJ & $1(1)$ \\
\hline Gonzales Catan & MNHN & $1(1)$ \\
\hline \multirow[t]{2}{*}{ Hurlinghan, Ayo Moron } & MNRJ & $1(1)$ \\
\hline & MNHN & $1(1)$ \\
\hline Mar del Sur & MNHN & $1(1)$ \\
\hline Miramar & MNRJ & $1(1)$ \\
\hline Passage Talavera & MNHN & $2(2)$ \\
\hline Punta Lara & MNHN & $1(1)$ \\
\hline Pergamino & MNRJ & $1(1)$ \\
\hline Quiroga & MNHN & $2(2)$ \\
\hline \multirow{2}{*}{ Zarate } & MNRJ & $1(1)$ \\
\hline & MNHN & $1(1)$ \\
\hline \multicolumn{3}{|l|}{ ENTRE RIOS } \\
\hline Arroyo Sagastone, Km 143.800 * & MNRJ & $1(1)$ \\
\hline \multicolumn{3}{|l|}{ Grupo 4} \\
\hline \multicolumn{3}{|l|}{ URUGUAI } \\
\hline \multicolumn{3}{|l|}{ Canelones } \\
\hline Bañado Tropa Vieja & MNHN & $11(11)$ \\
\hline Carrasco & MNHN & $1(1)$ \\
\hline Arryo las Bruxas & MNHN & $1(1)$ \\
\hline \multicolumn{3}{|l|}{ Rocha } \\
\hline Camino del Indio & MNHN & $1(1)$ \\
\hline \multicolumn{3}{|l|}{ Artigas } \\
\hline San Gregorio & MNHN & $1(1)$ \\
\hline \multicolumn{3}{|l|}{ Montevideo } \\
\hline Cerro & MNHN & $1(1)$ \\
\hline Carrasco & MNHN & $1(1)$ \\
\hline
\end{tabular}

*. Localidade sem indicação na figura 2.

Nas diversas instituições visitadas obteve-se informações de um total de 94 exemplares de 37 diferentes localidades. $\mathrm{O}$ total de crânios de animais adultos com procedência e sexados foi de 46 e 55 peles (Fig. 2).

Adicionaram-se à amostra exemplares do Museu Nacional de História Natural de Montevideo-Uruguai num total de 27 crânios da Argentina e Uruguai, cujas medidas encontram-se publicadas em XIMENEZ (1967) e tiveram por critério crânios que apresentavam dentição definitiva.

Foram tomadas as seguintes medidas craniométricas (Fig. 1), utilizando-se paquímetro de precisão de $0,05 \mathrm{~mm}$ :

1) Comprimento côndilo basal (CCB): distância do ponto mais anterior do crânio na interseção pré-maxilar com o ponto mais posterior do côndilo occipital; 2) Comprimento basal (CB): distância do ponto mais anterior do crânio na interseção pré-maxilar com a base do foramem magnum no plano sagital; 3) Comprimento palatal $(\mathrm{CP})$ : distância da interseção anterior do pré-maxilar até o 


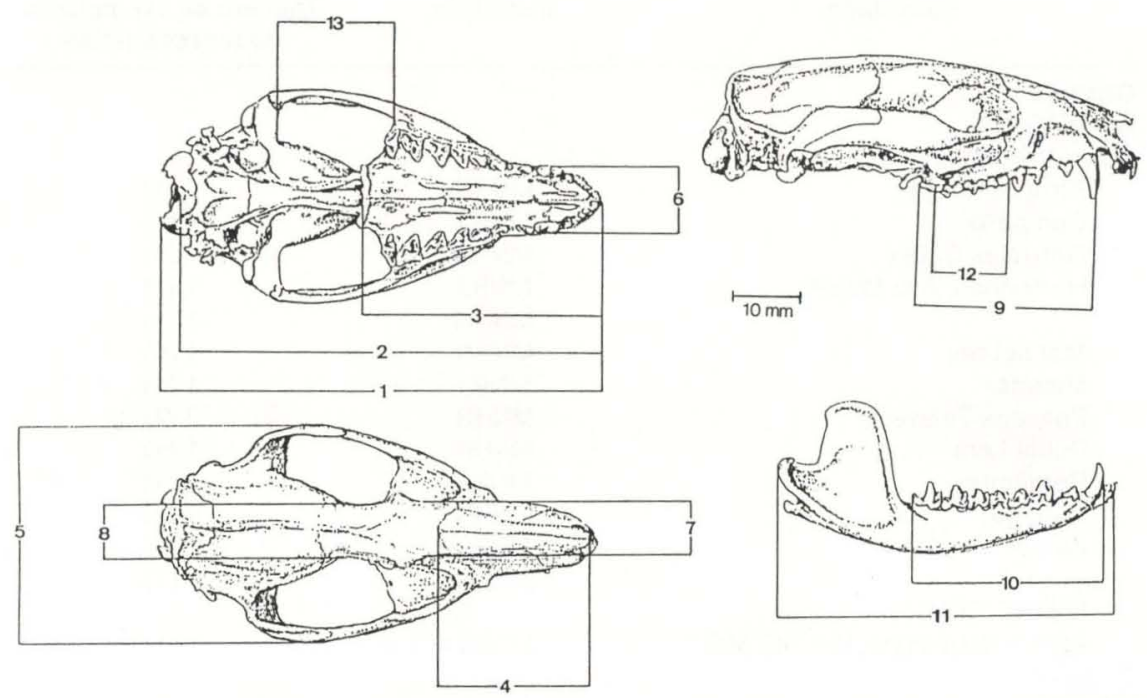

Fig. 1. Crânio de Lutreolina crasicaudata em vista dorsal, ventral e lateral de crânio e mandibula, assinalando as medidas utilizadas [Modificado de J.P. Lufkin apud MARSHALL (1978)].

ponto mais posterior do palato; 4) Comprimento máximo da sutura nasal (CSN): ponto mais anterior da sutura dos nasais até o ponto mais posterior da mesma; 5) Largura do arco zigomático máxima (LAZ): distância entre os pontos mais salientes do arco zigomático; 6) Largura do rostro máxima (LRM): distância entre os pontos mais externos dos bordos alveolares dos caninos superiores; 7) Largura inter orbitária mínima (LIO): menor distância entre as bordas das fossas orbitais; 8) Largura pós-orbitária mínima (LPM): largura mínima do crânio a nível do estreitamento pós orbitário. 9- Comprimento alveolar da série dentária superior (SDS): distância entre o ponto mais anterior da borda alveolar do canino superior até o ponto mais posterior da borda alveolar do último molar; 10) Comprimento alveolar da série dentaria inferior (SDI): distância entre o ponto mais anterior da borda alveolar do canino inferior até o ponto mais posterior da borda alveolar do último molar; 11) Comprimento máximo da mandíbula (CMM): distância entre o ponto mais anterior da mandíbula e o ponto mais posterior do côndilo articular esquerdo; 12) Comprimento M1-3 (CM): distância entre a borda alveolar anterior do primeiro molar e o borda alveolar posterior do terceiro molar; 13) Diâmetro da fossa temporal (DFT): maior diâmetro da fossa temporal.

De acordo com a região de procedência (Tab. I), foram formados os grupos que seguem para verificar se existiam diferenças entre os mesmos: Região sudeste do Brasil (Grupo 1); Região sul do Brasil (Grupo 2); Argentina (Grupo 3); Uruguai (Grupo 4). 


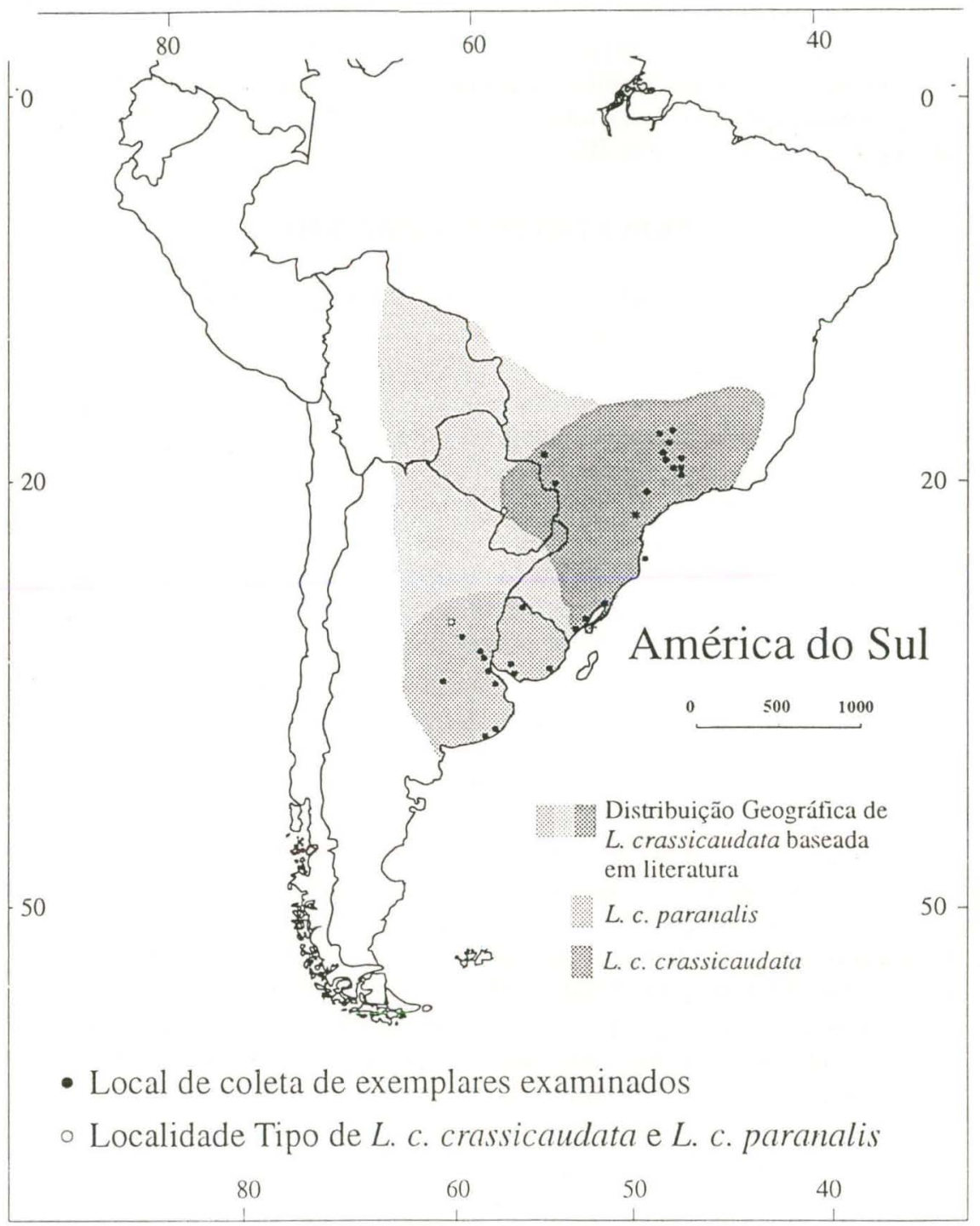

Fig. 2. Distribuição de Lutreolina crassicaudata segundo MARSHALL (1978) e de L. c. crassicaudata e L. c. paranalis a partir dos locais de coleta de exemplares examinados e localidade tipo das duas subespécies. 
As medidas craniométricas destes grupos foram submetidas a análise de variância com dois fatores: procedência e sexo. O teste de Scheffé foi utilizado para a comparação múltipla de médias de cada procedência e sexo (STEEL \& TORRIE 1980).

A separação de médias neste teste ocorreu quando a probabilidade de diferença significativa entre grupos ou sexo era superior a $95 \%$. A probabilidade do teste identificar erroneamente diferenças significativas entre populações iguais, mas com diferenças apenas nos indivíduos amostrados (erro tipo I) também foi calculado com o parâmetro "P" (Tab. II, III).

\section{RESULTADOS E DISCUSSÃO}

Lutreolina crassicaudata parece estar restrita a áreas de formações herbácea-arbustiva e matas de galerias, sendo comumente encontrada em regiões alteradas ou mesmo urbanizadas.

O comprimento de corpo para exemplares adultos examinados variaram de 234,5mm (602-UFSC, fêmea) a 340mm (077-UFSC, macho com a cauda cortada) e a cauda variando de $228,5 \mathrm{~mm}$ (602-UFSC) a $305 \mathrm{~mm}$ (24450-MNRJ, macho), sendo o peso de 246 gramas (601-UFSC, fềmea) a 793 gramas (077-UFSC).

A análise de variância confirma a hipótese de diferenças entre as médias dos grupos para as medidas CCB, LAZ, SDS, SDI, CMM e CM. As medidas CCB, LAZ, SDS e SDI separam os grupos 1 e 2 dos grupos 3 e 4 ( $P<0,0001)$. Da mesma forma a medida CMM $(\mathrm{P}=0,0006)$. A medida $\mathrm{CM}$ separou o grupo 3 dos demais com $\mathrm{P}<0,0001$ (Tab. II).

O teste de Scheffé evidencia a separação dos dados de animais coletados no Brasil daqueles da Argentina e Uruguai para as medidas CCB, LAZ, SDS, SDI e $\mathrm{CMM}$, apresentando alto nível de significância $(\mathrm{P}<0,0006$, Tab. II), demonstrando um maior tamanho de crânio dos animais da Argentina e Uruguai.

No grupo da Argentina, as sete medidas craniométricas que não diferenciaram, possuiam entre cinco e seis indivíduos na amostra. As mesmas medidas no grupo do Uruguai não possuiam exemplares. Possivelmente a amostra para estas sete medidas são insuficientes e necessitam de maior critério quanto a determinação da idade para diferenciar os grupos do Brasil do grupo da Argentina.

Assim, os dados levantados neste estudo diferem da opinião de MARSHALL (1978) apontando para separação de, no mínimo, duas subespécies na região meridional da América do Sul (Fig. 2):

- Lutreolina crassicaudata crassicaudata, localidade tipo Assunção distribuindo-se ao leste do Paraguai, nordeste da Argentina, pelo menos em Misiones e no Brasil (sul do Mato Grosso do Sul, São Paulo, Paraná, Santa Catarina e Rio Grande do Sul) e;

- Lutreolina crassicaudata paranalis $\mathbf{s p p}$. rev., com localidade tipo em Las Rosas Província de Santa Fé, distribuindo-se na região oriental da Argentina, nas províncias de Santa Fé e Entre Rios até o sul de Buenos Aires e estendendo-se por todo o Uruguai. 
Tabela II. Análise das medidas craniométricas dos diferentes grupos da amostra. (n) Número de exemplares da amostra, $(\mathbf{P})$ probabilidade de erro tipo $\mathrm{I}$.

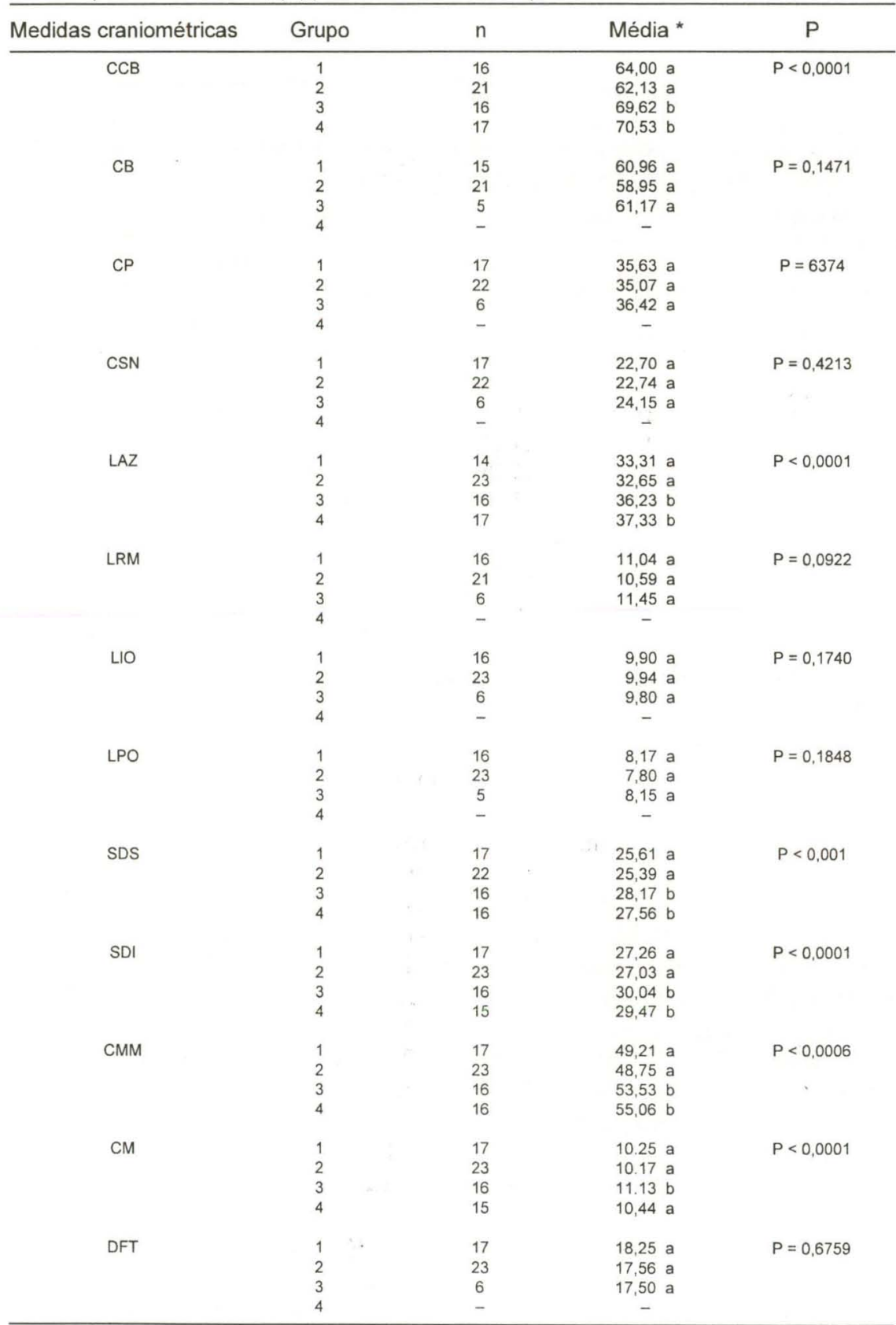

*. Médias seguidas por letras diferentes são significativamente diferentes (Scheffé 5\%). 
As medidas craniométricas demonstram claro dimorfismo sexual para as média de 10 das 13 medidas (Tab. III).

A largura inter orbitária mínima (LIO), a largura pós orbitária mínima (LPO) e o diâmetro da fossa temporal (DFT) também foram superiores em machos apesar de não se apresentarem significativamente diferentes a nível de 5\% (Tab. III).

Tabela III. Análise das medidas craniométricas de machos e fêmeas da amostra. (n) Número de exemplares na amostra, $(\mathrm{P})$ probabilidade de erro tipo I.

\begin{tabular}{cccccc}
\hline \multirow{2}{*}{$\begin{array}{c}\text { Medidas } \\
\text { craniométricas }\end{array}$} & \multicolumn{2}{c}{ Machos } & \multicolumn{2}{c}{ Fêmeas } & $\mathrm{P}$ \\
\cline { 2 - 6 } & $\mathrm{n}$ & Médias * & $\mathrm{n}$ & Médias & \\
\hline CCB & 41 & $69,28 \mathrm{a}$ & 29 & $62,12 \mathrm{~b}$ & $\mathrm{P}<0,0000$ \\
CB & 22 & $62,35 \mathrm{a}$ & 19 & $57,72 \mathrm{~b}$ & $\mathrm{P}<0,0020$ \\
CP & 24 & $36,50 \mathrm{a}$ & 21 & $34,29 \mathrm{~b}$ & $\mathrm{P}<0,0068$ \\
CSN & 23 & $23,77 \mathrm{a}$ & 22 & $22,03 \mathrm{~b}$ & $\mathrm{P}<0,0092$ \\
LAZ & 41 & $36,23 \mathrm{a}$ & 29 & $32,64 \mathrm{~b}$ & $\mathrm{P}<0,0000$ \\
LRM & 23 & $11,20 \mathrm{a}$ & 20 & $10,51 \mathrm{~b}$ & $\mathrm{P}<0,0159$ \\
LIO & 24 & $10,05 \mathrm{a}$ & 21 & $9,75 \mathrm{a}$ & $\mathrm{P}<0,2349$ \\
LPO & 24 & $8,15 \mathrm{a}$ & 20 & $7,77 \mathrm{a}$ & $\mathrm{P}<0,1206$ \\
SDS & 42 & $27,47 \mathrm{a}$ & 29 & $25,24 \mathrm{~b}$ & $\mathrm{P}<0,0000$ \\
SDI & 42 & $29,36 \mathrm{a}$ & 29 & $26,73 \mathrm{~b}$ & $\mathrm{P}<0,0000$ \\
CMM & 42 & $53,77 \mathrm{a}$ & 30 & $47,91 \mathrm{~b}$ & $\mathrm{P}<0,0000$ \\
CM & 42 & $10,67 \mathrm{a}$ & 29 & $10,17 \mathrm{~b}$ & $\mathrm{P}<0,0006$ \\
DFT & 24 & $18,37 \mathrm{a}$ & 22 & $17,20 \mathrm{a}$ & $\mathrm{P}<0,0789$ \\
\hline
\end{tabular}

Médias na mesma linha seguidas por letras diferentes são significativamente diferentes (Scheffé $5 \%$ ).

Das peles analisadas, 42 apresentaram coloração dorsal castanha com pequenas variações no padrão de tonalidade. A coloração ventral mostrou-se mais uniforme, com tonalidade amarela-clara. No Museu de História Natural da Universidade Estadual de Campinas (UNICAMP) foi possível perceber variações de coloração, sendo a região dorsal mais escura nos exemplares número 1350-ZUEC, 1351-ZUEC, 1471-ZUEC, 1536-ZUEC coletados na cidade de Campinas, São Paulo, e 1499-ZUEC no município de Américo Brasiliense, São Paulo. Nas peles do Museu Nacional do Rio de Janeiro, o exemplar de número 24161-MNRJ com procedência de Campinas apresentou padrão semelhante àqueles mais escuros observados na UNICAMP. Os exemplares 24447-MNRJ, 24448-MNRJ, 24449MNRJ, 24450-MNRJ, 24451-MNRJ e 24452-MNRJ, procedentes da Argentina apresentavam coloração geral amarela clara. Um exemplar procedente de São Lourenço, Rio Grande do Sul, de número 24461-MNRJ, com coloração marrom escura tanto no dorso quanto no ventre destacava-se dos demais pela má conservação e coloração bastante mais escura.

Nas observações realizadas no Museu de História Natural da UNICAMP e no Museu Nacional, Rio de Janeiro, foi possível perceber que peles com uma mesma procedência (Campinas) apresentavam padrões de coloração diferentes, com algumas apresentando o dorso mais escuro que outras. Nas peles da coleção da UFSC 
foram observadas somente pequenas variações de coloração. No entanto todas as peles com procedência do sudeste e sul apresentaram como padrão de coloração predominante o castanho, com o ventre amarelo claro.

Quando vivos, os animais coletados no decorrer deste trabalho apresentavam coloração alaranjada no ventre, que após a preparação e permanência na coleção sofreram grande descoloração nesta região. A coloração castanha de peles recém taxidermizadas era mais viva, no entanto semelhante àquelas com vários anos na coleção.

Os resultados levantados neste estudo em coleções de referência comprovam as diferenças na coloração de animais de uma mesma procedência. O padrão de coloração da pelagem segundo MONTEIRO FILHO \& DIAS (1990) varia em diferentes épocas do ano e até mesmo em pequenos intervalos de tempo.

Foi observado após o manuseio de um exemplar com coloração castanho avermelhada coletado na Ilha de Santa Catarina o tingimento das mãos, tendo sido difícil a retirada desta coloração, fato este também observado por MONTEIRO FILHO \& DIAS (1990).

O exemplar de número 244661-MNRJ de São Lourenço apresentou coloração marrom escura muito diferenciada das outras peles observadas, o que poderia evidenciar a impossibilidade de se basear na coloração da pelagem para estudos de sistemática, mas tratava-se de um exemplar único e que se apresentava em estado de conservação bastante comprometido.

O uso da coloração como critério taxonômico é criticado por CABRERA (1957) e XIMENEZ (1967), uma vez que animais de mesma procedência apresentam variações individuais na coloração. Para estes dois autores, qualquer conclusão quanto à sistemática destes marsupiais baseada em exames de exemplares de coleções de museus está exposta a graves erros, no entanto a homogeneidade de cores dos seis exemplares procedentes da Argentina (no MNRJ) sugere a possibilidade da manutenção da subespécie $L$. c . bonaria Thomas, 1923 que tem por localidade tipo Los Yngleses, Ajo, na Província de Buenos Aires. Estudos futuros com maior número de exemplares desta região poderão esclarecer esta questão.

Vários autores afirmam que machos de L. crassicandata tendem a ser maiores que fêmeas (CABRERA \& YEPES 1960; XIMENEZ 1967; MONTEIRo FiLHO \& DIAS 1990), o que foi constatado para a média de todas as medidas craniométricas utilizadas na amostra, sendo 10 das 13 medidas significativamentes diferentes a nível de $5 \%$, demonstrando assim o dimorfismo sexual para medidas craniométricas da espécie.

A análise de variância demonstrou que cinco das 13 medidas que possuiam um número razoável de exemplares na amostra apresentaram-se significativamente diferentes a nível de 5\%. Desta forma, entende-se que a subespécie $L$. c paranalis é válida.

AGRADECIMENTOS. Aos curadores Ana Maria Malva Ramos Costa (MZUSP), Luiz Flamarion Barbosa de Oliveira (MNRJ), Emygdio Leite de A. Monteiro Filho (ZUEC), Vanessa Person (MHNCI) e Marta E. Fabian (FZBRS). Ao Prof. Paulo Ogliari e Marcelo Mazzolli pela 
participação na banca de defesa do trabalho de conclusão do Curso de Biologia que foi base para este trabalho. À Sérgio Althoffe Claudia Leandro pelo auxílio em coletas de dados e campo e Eduardo Faria pelo auxilio na montagem das figuras deste trabalho. Ao Dr. Paulo César Simões-Lopes pelo apoio nas coletas de dados e revisões de texto. Ao Diretor do Centro de Ciências Biológicas (UFSC), Prof. Carlos Miguez pelo auxílio em passagens para deslocamento aos Museus.

\section{REFERÊNCIAS BIBLIOGRÁFICAS}

Cabrera, A. 1957. Catálogo de los Mamíferos de América del Sur. Revta Mus. Arg. Cien. Nat.. 4 (1): 1-307.

Cabrera, A. \& J. Yepes. 1960. Mamiferos Sud Americanos. Buenos Aires, Dep. Public. Cient. Argent., vol. 1, 187p.

Desmarest, A.G. 1804. Tableau méthodique des mammiferes, p. 5-38. In: Nouveau dictionnaire d'histoire naturelle. Paris, vol. 24.

Marshall, L.G. 1978. Lutreolina crassicaudata. Mammalian Species 91: 1-4.

Monteiro Filho, E. L. DE A. \& V. SouzA DiAs. 1990. Observações sobre a biologia de Lutreolina crassicaudata (Mammalia, Marsupialia). Rev. Bras. Biol. 50 (2): 393-399.

NOWAK, R.M. \& J.C. PARADISO. 1983. Walkers mammals of the world. Baltimore, John Hopkins Univ. Press, vol. I, $4^{\text {th }}$ ed., 568p.

SteEl, R.G.D. \& J.H. TORRIE. 1980. Principles and Procedures of Statistics - a biometrical approach. New York, McGraw Hill Publishing Company, $2^{\text {nd }}$ ed., $\mathrm{XXI}+633 \mathrm{p}$.

Thomas, O. 1923. Geographical races of Lutreolina crassicaudata. Ann. Mag. Nat. Hist. 11 (9): 583-585.

XIMENEZ, A. 1967. Contribuicion al conocimiento de Lutreolina crassicaudata (Desmarest, 1804) y sus formas geograficas (Mammalia - Didelphidae). Comunicaciones Zoologicas del Museo de Historia Natural de Montevideo 9 (112):1-7.

Recebido em 08.V.1996; aceito em 22,XI.1996. 